\title{
Abnormal nonstoring capillary endothelium: a novel feature of Gaucher disease. Ultrastructural study of dermal capillaries
}

\author{
Helena Hůlková • Helena Poupětová • Klaus Harzer • \\ Pramod Mistry • Johannes M. F. G. Aerts • \\ Milan Elleder
}

Received: 28 July 2009 /Revised: 30 October 2009 /Accepted: 10 November 2009/Published online: 5 January 2010

(C) The Author(s) 2009. This article is published with open access at Springerlink.com

\begin{abstract}
Ultrastructural study of skin biopsies in two cases of Gaucher disease (GD) patients (types II and III) revealed hitherto unknown alteration of the blood capillary endothelial cells (ECs) featured by hypertrophy and numerous subplasmalemmal microvesicles underneath both the apical and basal membranes. There was also prominent apical membrane folding with formation of filiform and large cytoplasmic projections, with occasional transcapillary cytoplasmic bridges. Similar, though less frequently expressed, changes were manifested at the basal membrane by numerous cytoplasmic projections into the subendothelial space. Regressive changes with EC breakdown were rare. Lysosomal storage was always absent. Besides EC hypertrophy, there was also increased EC density in the
\end{abstract}

Communicated by: Gregory Pastores

H. Hůlková $\cdot$ H. Poupětová $\cdot$ M. Elleder $(\square)$

1st Faculty of Medicine and General Teaching Hospital, Institute of Inherited Metabolic Disorders,

Charles University in Prague,

Ke Karlovu 2, Bldg. D,

12000 Praha 2, Czech Republic

e-mail: melleder@cesnet.cz

\section{K. Harzer}

Department of Pediatrics and Child Development, Universitäts-Kinderklinik,

Tübingen, Germany

P. Mistry

Section of Pediatric Hepatology and Gastroenterology,

Department of Pediatrics and Medicine, Yale School of Medicine,

New Haven, CT, USA

J. M. F. G. Aerts

Department of Medical Biochemistry, Academic Medical Center,

University of Amsterdam,

1105 AZ Amsterdam, The Netherlands capillary lumen, leading to pronounced changes in capillary architecture with loose or incomplete EC anchoring. There were also signs of EC sprouting. Some pericytes displayed an increase in size and number of cytoplasmic processes, which often extended into distant pericapillary regions. The spectrum of changes suggests that a significant positive growth effect on EC occurs in GD. The putative mechanisms triggered by GBA1 deficiency leading to EC involvement are discussed. The authors are well aware of the fact the results, based on a nontraditional type of bioptic samples, are preliminary, but they are worth following, as further ultrastructural and functional studies of blood endothelium in GD may open a novel field in molecular cell pathophysiology of the disorder: endothelial dysfunction.

\section{Introduction}

Gaucher disease (GD) was first described in 1882 by a young medical student. Molecular delineation commenced in 1934 with identification of the nature of stored lipid, followed 31 years later by recognition of metabolic defect in the form of inherited deficiency of glucocerebrosidase (GCase), and 16 year later by characterization of the GBAl gene and description of the first disease mutations. Many of these milestones are elegantly described in a monograph edited at the occasion of centennial anniversary of the description of the disorder (Desnick et al. 1982). Knowledge of cellular pathology of this most frequent lysosomal enzymopathy is generally viewed as storage restricted to macrophages transformed into cytologically unique Gaucher cells (GC) characterized by active secretion of numerous molecules, some of which are in use as biomarkers in clinical practice (Aerts and Hollak 1997; Boven et al. 2004; Cox 2005; Deegan et al. 2005). Lysosomal storage in other cell types has never been 
reported. Therefore, the pathological focus has been on the pattern of GC infiltration in different organs. Interestingly, neurodegeneration, a feature of GD type II, is not associated with lysosomal storage and is believed to involve direct toxic effects of glucosylsphingosine on neuronal cells. The current understanding of the pathology of GC is described comprehensively in excellent monographs (Beutler and Grabowski 2001; Lee 1982; Lee 2007). However, there are significant gaps in our understanding of the pathophysiologic basis of GD and cell types involved. Notably, there is a discrepancy between generalized enzymopathy that occurs in GD and restriction of lipid storage structures to one cell type, which led one of us to propose a hypothesis that might bridge the persisting gap between the firmly established molecular definition and the loosely defined cell pathology (Elleder 2006). In this report, we present for the first time prominent alteration of the capillary endothelial cells (ECs) disclosed by routine ultrastructural examination of skin biopsies of patients with type II and type III GD. To the best of our knowledge, we are not aware of any study of ECs in GD, despite the voluminous literature (see above) and recent report (Chen and Wang 2008). Reports on skin pathology are limited to clinical observations in GD I (Goldblatt and Beighton 1984). Reports on all types of early-onset GD focused on epidermal abnormalities (Holleran et al. 1994a, b, 2006; Reissner et al. 1998; Sharma et al. 2000; Sidransky et al. 1992) or general histology (Eblan et al. 2005; Mignot et al. 2003; Sherer et al. 1993; Spear et al. 2007; Stone et al. 1999; Van Gysel et al. 2002). Also, reports on mouse models of GD did not mention EC status. Our findings of dermal capillaries point to a novel aspect of pathology of GD: remarkably intensive alterations of blood capillary endothelium suggesting EC overstimulation. This prompts consideration of the presence of a novel aspect of the pathophysiology of GD, i.e., that of endothelial dysfunction and its implications in several mechanisms of GD, such as a vascular osteonecrosis and pulmonary vascular disease.

\section{Material and methods}

\section{Patients}

Case 1 A male infant presented with severe hepatosplenomegaly, psychomotor retardation associated hypertonia, and tendency to opisthotonus. Interestingly, the child exhibited discrete reddish cutaneous maculae. Skin biopsy was taken at the age of 10 months. He died at the age of 13 months. Autopsy was not performed. Glucocerebrosidase (GCase) activity in white blood cells (WBC) was $1.7 \mathrm{nmol} / \mathrm{mg}$ protein/h [controls $6.5-16.9$, mean \pm standard deviation (SD) $9.34 \pm 1.75, n=113$ ); in cultured skin fibroblasts $6 \mathrm{nmol} / \mathrm{mg}$ protein $/ \mathrm{h}$ (controls $154-536$, mean \pm SD $322 \pm$
119, $n=15)$. Sequencing the GBA1 gene revealed the patient to be compound heterozygote for L444P and S107L mutations (Hodanova et al. 1999). Chitotriosidase activity in plasma was not determined. The patient was classified as having type II GD.

Case 2 A boy aged 2 years presented with prominent hepatosplenomegaly, pancytopenia, and minimal neurological involvement manifesting as mild oculomotor symptoms with slowed, hypometric, horizontal saccades and suspected convergent strabismus. Skin was grossly normal without any efflorescences. Skin biopsy was taken at the age of 21 months. GCase activity in cultured skin fibroblasts was $13.6 \mathrm{nmol} / \mathrm{mg}$ protein $/ \mathrm{h}$ ( $4.2 \%$ of control mean value). GBA1 gene analysis revealed the patient to be homozygous for L444P mutation. Chitotriosidase activity in plasma was $16,065 \mathrm{nmol} / \mathrm{ml}$ per hour (normal value $<80$ ). The patient's presentation and phenotype was consistent with type III GD.

Methods

Skin biopsies were taken for diagnostic purposes after informed consent was given the by patients' mothers in accordance with the Declaration of Helsinki. Skin biopsies, taken from the medial side of the upper arm of both patients were fixed in $4 \%$ paraformaldehyde (case 1) or $2 \%$ glutaraldehyde (case 2), embedded using standard processing procedures into an Epon-Araldite mixture, double stained, and examined using a Tesla 500 electron microscope and a Jeol 1200 EX. Endothelial ultrastructure was compared with series of skin biopsies of patients without diagnosis of lysosomal storage disorders.

GCase activity was determined in leukocytes and fibroblasts as described by Wenger and Williams (1991) using the artificial fluorogenic substrate 4-methylumbelliferyl- $\beta$-Dglucopyranoside (Sigma M 3633). Protein concentration was determined by the method of Hartree (Hartree 1972). Enzyme analysis was done in the same laboratory.

\section{Results}

Ultrastructure of dermal capillaries

Dermal capillaries were not generally increased in number. However, they were frequently found to be in clusters. The general capillary architecture had frequent and significant alterations, with increased size and irregular contours. In longitudinally oriented sections, the tortuosity was very apparent (Fig. 1a, b). The number of ECs was increased (4-6/capillary) compared with controls (2-3 ECs/capillary). 

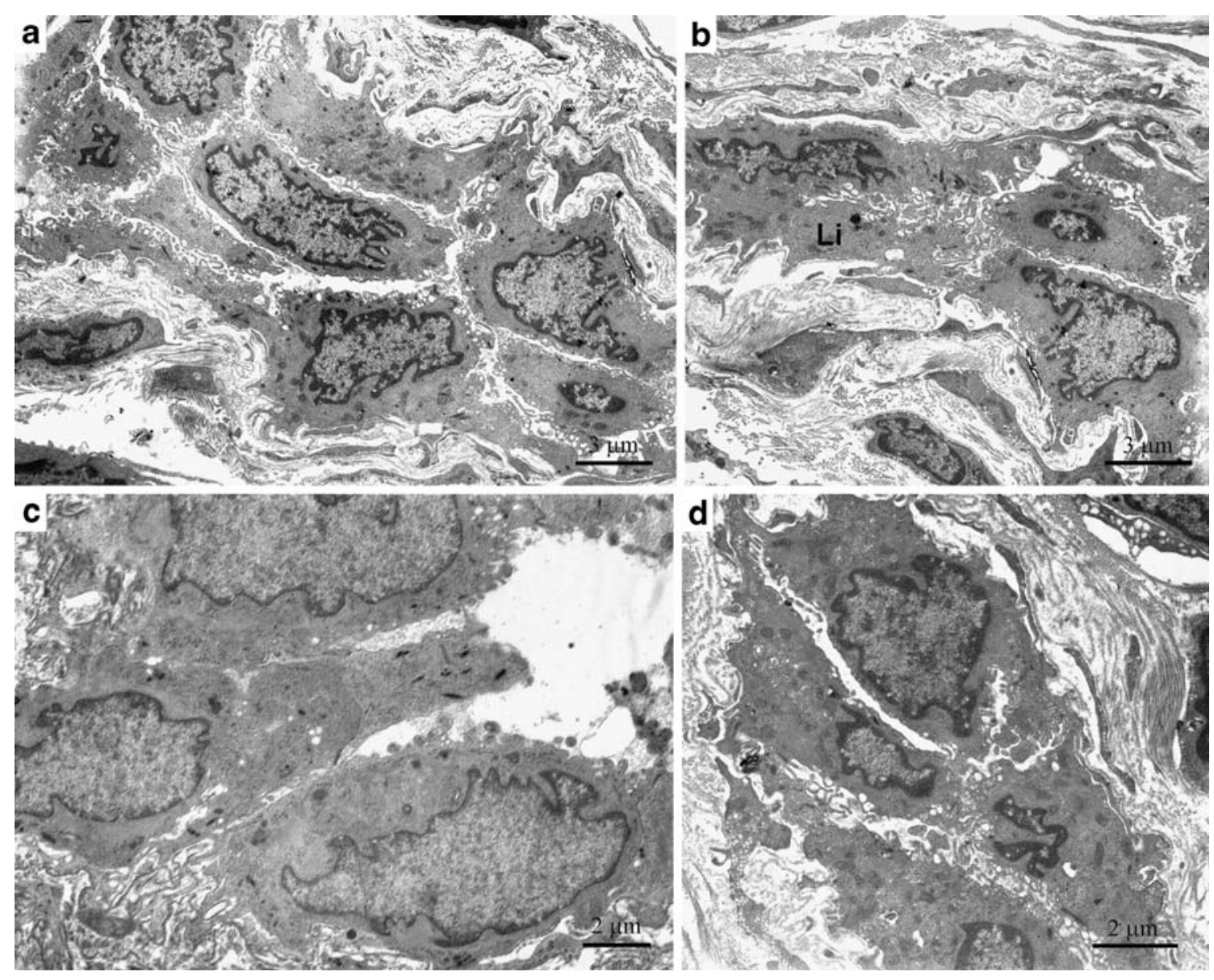

Fig. 1 a Dermal capillary (case 2) showing numerous enlarged endothelial cells (ECs) with prominent folding of both the apical and basal pole membranes. b Dermal capillary (case 2) showing enlarged, irregular ECs with uneven apical pole membranes. One of them can be seen to be loosely anchored. Li lipopigment deposits. c Dermal capillary (case 1) showing enlarged ECs with prominent folding at the

EC size was clearly increased and the cytology altered. EC enlargement was substantial, resulting in a marked reduction or obstruction of the capillary lumen (Fig. 1d, 2c). In many capillaries, EC apical pole protruded frequently into existing lumina through polyp-like projections (Fig. 2c, d). Occasionally, there were cytoplasmic bridges extending across the capillary lumen (Fig. 2b). In some capillaries with higher EC densities, ECs displayed loose or incomplete intercellular connections, resulting in decreased EC anchoring (Fig. 1a, b, 2a).

The expanded EC cytoplasm contained variable numbers of Weibel-Palade granules of various sizes, as well as clusters of typical mitochondria with relatively dense innermembrane cristae. Frequently, there were thick bands of intermediate filaments $(8.4 \mathrm{~nm}$ in diameter). Lysosomal storage was never observed. Nonspecific, discrete lipofuscin granules were exceptional. Rarely, there were typical small multivesicular bodies. The Golgi endoplasmic reticulum complex was generally present, and the external nuclear basal pole and limited by a multilayered basement membrane. ECs display loose intercellular connections. The apical part of the EC at the center protrudes prominently into the capillary lumen. d Dermal capillary (case 2) with an obliterated lumen caused by enlarged ECs. Note the prominent folding of the apical membranes and fusion between opposite ECs. Note also the loose intercellular connections

lamina was easily discernible. Numerous cytoplasmic ribosomes $(\approx 25 \mathrm{~nm}$ in diameter $)$ were present in all cases. There were numerous subplasmalemmal vesicles resembling caveolae $(\approx 83 \mathrm{~nm}$ in diameter) beneath both the apical and basolateral poles and accompanied by larger microvesicles (up to $170 \mathrm{~nm}$ in diameter) deeper in the cytoplasm. The nuclei were enlarged with irregular contours, frequently cleaved or multilobed, with slender junctional segments and rich in euchromatin. Condensed chromatin was present only at the nuclear periphery. Mitosis was not seen. The apical poles of the ECs frequently displayed an enormous degree of irregularity, with many folds of various sizes, thickness, and density or with many discrete filiform-type projections protruding noticeably into the capillary lumen. They contained mainly ribosomes, infrequently admixed with occasional microvesicles, and exceptionally with Weibel-Palade granules (Fig. 1d, 2d). In some ECs, the apical cytoplasm protruded deeply into the capillary lumen (Fig. 1c). 

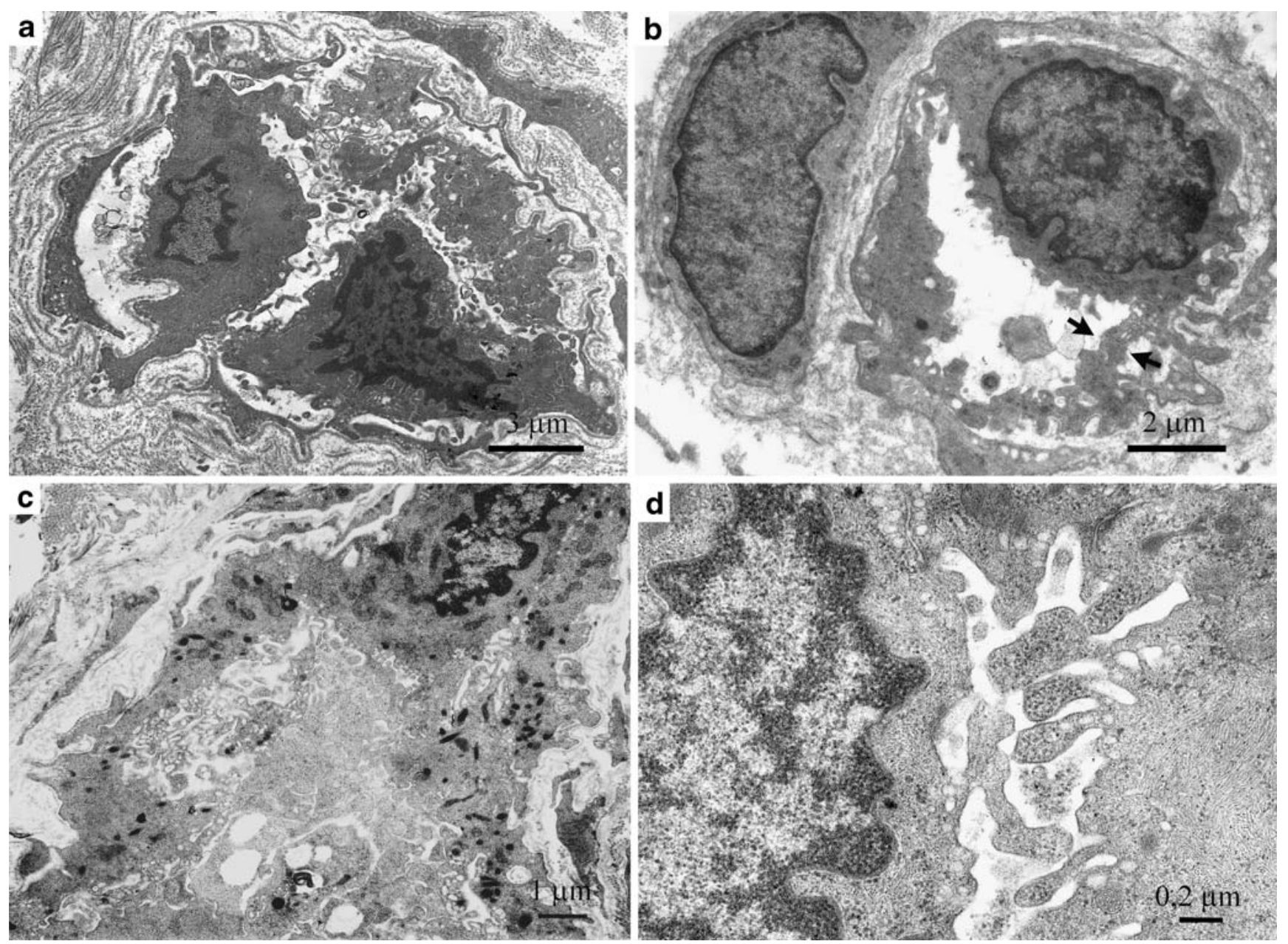

Fig. 2 a Dermal capillary (case 2) showing discontinuous endothelial cells (ECs) displaying loose anchoring and formation of a second endothelial layer. b Dermal capillary (case 1) with an enlarged single EC. A prominently activated apical pole forms a transluminal bridge (arrows). Note the large pericyte on the left. c Dermal capillary (case 2) with the lumen almost completely obliterated by the enlarged,

The basal pole was serrated and frequently folded with cytoplasmic, foot-like extensions, projecting into the pericapillary space, forming a fragmentary or complete second endothelial layer (Fig. 2a). Each of the extensions displayed a basement membrane on its external surface. Basement membranes were generally thin and frequently multiple and admixed with the aforementioned basal EC extensions. Occasionally, the EC basement membranes were barely detectable, especially underneath prominently enlarged ECs (Fig. 2c). Degenerative changes were exceptional but were occasionally observed in individual ECs (Fig. 3a).

There were recurrent findings suggestive of angiogenesis, i.e., sprouting of tip-like EC or tube-like structures with primitive lumina lined by less differentiated ECs. In some cases, the cytoplasm was free of nuclei (Fig. 3c). Pericytes were observed to participate in the process. Process compatible with intussusceptive type angiogenesis was also folded EC cytoplasm. Note the cytoplasmic transformation into a dense mesh and the numerous Weibel-Pallade granules. The thin basement membrane is almost invisible on the left side. d Dermal capillary (case 2) showing details of EC apical membrane folding together with numerous ribosomes and occasional microvesicles

observed. ECs protruding into the capillary lumina had nuclei situated within the luminal part (Fig. 3d). Capillaries with near-normal ECs were infrequent. Their cytoplasm was narrow and less differentiated.

Some pericytes were significantly enlarged. Frequently, the space between the pericyte inner aspect and EC outer aspect was wider, without any sign of focal gap-junction connections, suggesting abnormal fluid accumulation (perhaps caused by leaky capillaries). There were often contacts between slender EC projections and pericyte cytoplasm (never direct fusion). Pericyte cytoplasm was rich in caveolae, which were distributed along the cell membrane. Some pericytes were completely detached and situated a considerable distance from the capillary, without any sign of cytoplasmic connections (as seen in plain section). Despite the detachment, they were completely surrounded by a pericellular membrane and shared cytoplasmic features 

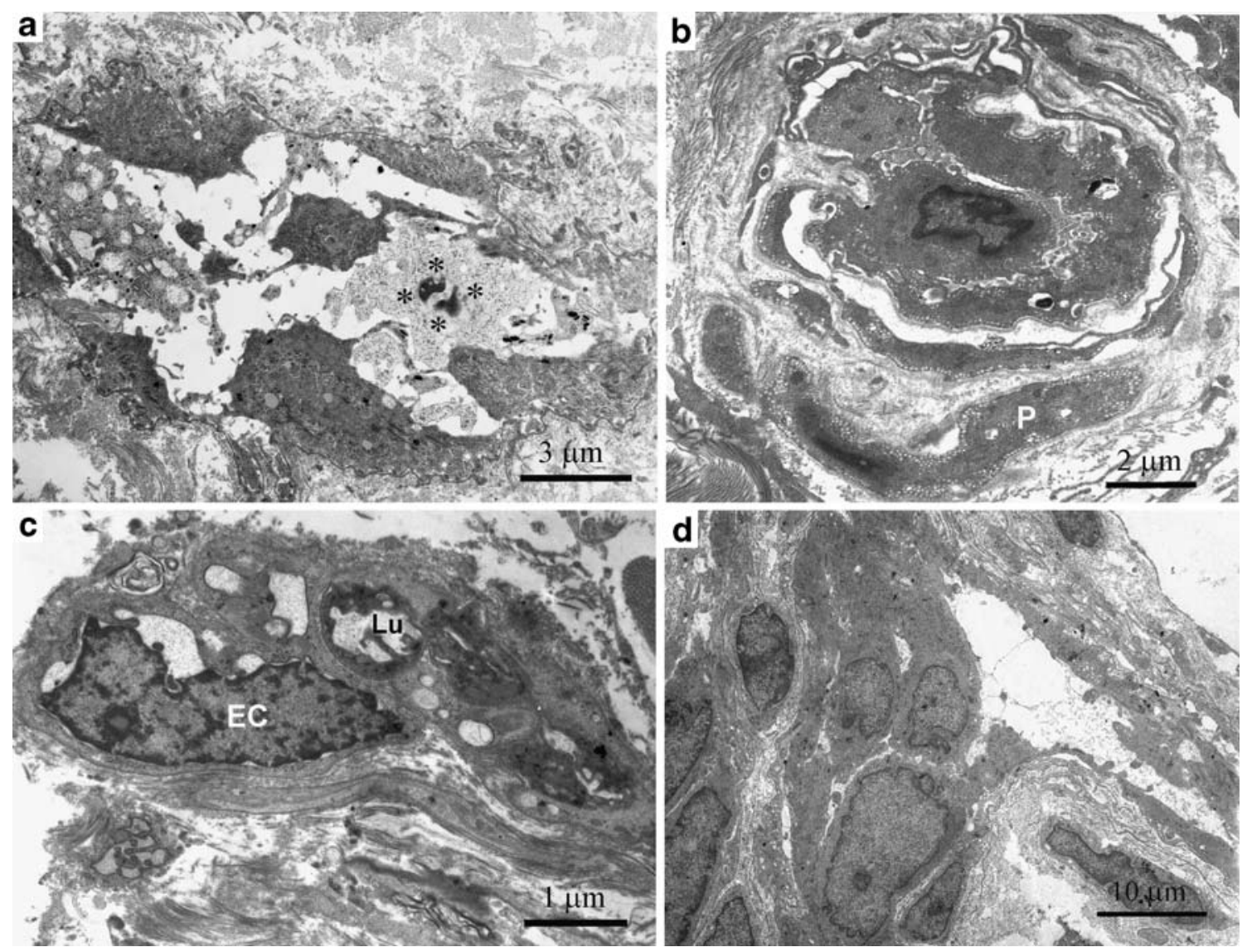

Fig. 3 a Dermal capillary (case 1) showing a level of degeneration approaching cytoplasmic lysis (asterisks). b Dermal capillary (case 2) showing endothelial-cell (EC) hypertrophy and numerous surrounding pericytes. Note that one of the pericytes $(P)$ is some distance from the capillary and completely detached but still expresses a pericellular membrane and subplasmalemmal microvesicles around its entire perimeter. c Dermis (case 1) showing structures compatible with EC

with classically positioned pericytes (Fig. 3b, Fig. 4), being thus easily distinguishable from both individual dermal cell fibroblasts and perivascular macrophages. The latter were very rare and also never expressed any signs of typical glucocerebroside storage structures, only nonspecific residual bodies. No inflammatory changes were present. Capillary endothelium of arterioles and venules was present irregularly in the samples. Also, dermal lymphatic capillary endothelia could not be followed systematically. However, in many instances, both their nuclei and the perinuclear cytoplasm were enlarged, with many apical microvesicles.

\section{Other dermal structures}

Fibroblasts, mast cells, nerves, and smooth muscle cells (arrectores) were without recognizable structural alterations. Generally, there were numerous altered mitochondria in the excretory tubules of sweat glands. Similar structures are seen in skin biopsies from a variety of conditions and most sprouting. There is a single EC $(E C)$ with three cytoplasmic extensions, one of them definitely luminized $(L u)$. d Branched dermal capillary (case 1). Note the large lumen at the right lined with very thin EC cytoplasm. On the left are three enlarged ECs, which are prominently protruding into the obliterated lumen. The architectural pattern is compatible with intussusceptive-type angiogenesis

probably represent a normal stage of the mitochondrial life cycle (Carpenter 1988 and extensive personal experience). The secretory parts of the eccrine glands were present only in case 1 and were notable for their high degree of enlargement. In this sense, they differed significantly from skin eccrine glands at this age level (ME, unpublished observation).

\section{Comparative group}

In the comparative group of 300 skin biopsies of young children $(\approx 1-4$ years of age), EC changes were limited to enlargement of EC nuclei. Less frequently, there was enlargement of the cytoplasm with numerous WeibelPalade granules, abundant bundles of intermediate filaments, increased numbers of mitochondria, and occasional multivesicular bodies. Cell membranes at both the apical and basolateral poles were even, without any substantial irregularities, suggestive of activation. Multiple basement 

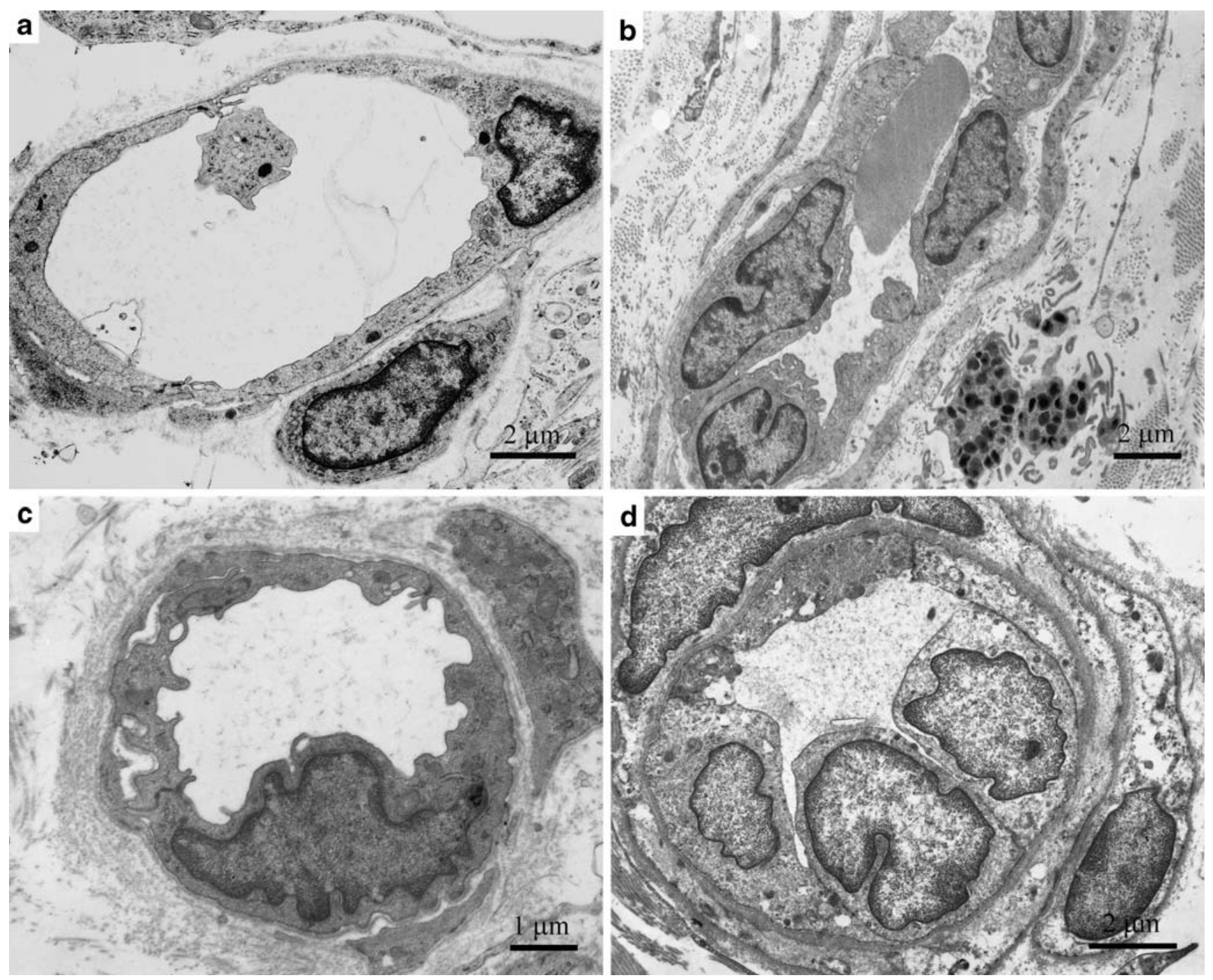

Fig. 4 Comparative group of skin biopsies showing variability of capillary endothelial cell (EC) appearance. a Flat EC, a frequent finding. b, c Larger EC with rudimentary folding of the apical membrane. d Enlarged EC with smooth membranes at both apical and basal poles

membranes and endothelial foot processes in the subendothelial space were scarcely seen. Pericytes, when present, were typically positioned, without any signs of systemic enlargement. Quite exceptionally, in three cases in which lysosomal storage, including GD, had been excluded, there were changes in some EC comparable with those seen in the case studies in this report.

\section{Discussion}

The spectrum of changes in the blood capillary endothelium described herein in GD points to classical hypertrophy represented by an enlarged nuclear compartment rich in euchromatin, increased cytoplasmic volume, and increased numbers of organelles, with the number of free ribosomes and amount of rough endoplasmic reticulum pointing to upregulation of proteosynthesis. These changes were accompanied by a prominent modulation of cell membranes, which suggests a considerable increase in their dynamics. It was expressed mainly in the luminal apical membranes, frequently to a remarkable degree, but also at the basal EC pole. The latter often displayed a highly serrated appearance and an increased size of the endothelial basal foot projections, which frequently created a second endothelial layer (Pavelka and Roth 2005). EC hypertrophy was accompanied by a process strongly resembling both types of angiogenesis (Burri and Djonov 2002; Djonov et al. 2003; Risau 1997): (a) intussusceptive (with various degrees of transcapillary pillar formation), and (b) sprouting type (with luminal expansions of various types). Regressive changes were rare and restricted to individual ECs. However, the presence of multiple basement membranes, resembling the situation in diabetes mellitus (Vracko and 
Benditt 1974), suggests increased turnover either of all ECs or turnover confined to basal pole processes. It should be stressed that there were no signs of glucocerebroside storage structures.

Taken together, there were signs of prominent EC hypertrophy, including a certain degree of angiogenic hyperplasia and focal regressive changes suggesting occurrence of a chronic stimulatory effect. EC changes resembled EC activation described in several experimental systems (AghaMajzoub et al. 2005; Dye et al. 2004; Fujimoto et al. 2004; Walski and Frontczak-Baniewicz 2003) or changes described in various spontaneous conditions such as adaptive capillary growth in overloaded rat skeletal muscles, (Zhou et al. 1998) inflammation (Polosukhin 1997), acute rejection of human renal allograft (Liptak et al. 2005), and other spontaneous conditions and experimentally induced states (Martin et al. 1980; Norrby 1997; Polverini 1995), which are generally classified as EC activation.

As these known causes of EC activation were absent in the two patients, we suggest two possible explanations of the phenomenon, both capable of exerting chronic EC activation effect. First, it is challenging to assess the direct role of enzymopathy. If enzymopathy plays a role in the development of the EC abnormalities described herein, the mechanisms a priori have to involve those other than accumulation of lysosomal glucocerebroside structures, as these are absent in this cell type. It should be kept in mind that the direct role of enzymopathy in type II GD results in neurodegeneration by pathways that appear not to involve lysosomal accumulation of glucocerebroside structures. It is of interest in this connection to point out that low-density lipoprotein (LDL) in patients with GD contains markedly elevated glucosylceramide and gangliosides, suggesting that many cell types, including ECs, are chronically supplied with increased amounts of glycosphingolipids destined for turnover (Ghauharali-van der Vlugt et al. 2008; Groener et al. 2008). Recently, one of us (Elleder 2006) proposed a more generalized involvement of diverse cells types in GD other than macrophages. In this paradigm, cell types other than macrophages are envisaged to have efficient transport mechanisms for accumulating glucocerebroside out of the lysosome were it becomes available as substrate for hydrolysis by neutral GCase (GBA2) (Boot et al. 2007; van Weely et al. 1993; Yildiz et al. 2006). Such a pathway may play a role in EC activation described herein via generation of ceramide and aberrant cell signaling thereof (Elleder 2006), similarly to what was described recently in studies of the mechanism of acute hypoxic pulmonary vasoconstriction (Cogolludo et al. 2009). Glucocerebroside itself may also have signaling activity, and its lysoproduct, glucosylsphingosine, also increased in GD, might contribute to EC cytotoxicity, activating cytokine imbalance. Recently, an ex vivo study in experimentally induced GC showed increase in GlcCer in microdomains (Hein et al. 2008), suggesting the possibility of their functional alteration.

Secondly, mechanisms underlying EC activation in GD could involve the role of cytokines and growth factors produced and secreted by activated macrophages as they transform into fully mature GCs (Cox 2005; Deegan et al. 2005; Hollak et al. 1997a; Michelakakis et al. 1996). This should be explored in the future by measureing cytokines and growth factors [i.e., vascular endothelial growth factor (VEGF)] in sera of GD patients, especially those who exhibit prominent vascular phenotypes such as pulmonary hypertension, hepatopulmonary syndrome, and splenic vascular lesions. In contrast to finding noted herein, in sarcoidosis, another disease involving macrophage activation, there are predominantly regressive changes of ECs (Planes et al. 1994; Tsukada et al. 1995) combined with inflammation and necrosis (Takemura et al. 1997). A picture of reactive angioendotheliomatosis with prominent endothelial capillary proliferation rarely seen in sarcoidosis (Shyong et al. 2002) was absent in our patients.

Taken together, the findings described herein strongly implicated EC activation and dysfunction in the pathophysiology of GD. The mechanisms responsible for EC transformation in GD can be most efficiently elucidated by exploring cultured ECs from GD patients and concurrent studies of newly developed authentic mouse models of the disease. Moreover, there is now the prospect of developing double-knock-out mouse models of $G B A 1$ and $G B A 2$ genes, which is especially pertinent to the concepts outlined herein. Such studies may challenge the macrophage-centric view of GD and help explain several features of the disease that currently defy explanation (Elleder 2006) i. e., tendency to pulmonary hypertension (Elstein et al. 2005) or permanent hypercoagulability and its consequences in GD (Adar et al. 2008; Elstein et al. 2000; Hollak et al. 1997b), as well as why patients with GD suffer from avascular osteonecrosis, when in fact there is evidence of increased vascularity (P. Mistry, unpublished observation). It is worth mentioning that recently advanced proteomics technology available to sensitively quantify plasma proteins revealed in GD patients marked abnormalities in coagulation and complement pathways that were partly corrected by enzyme replacement therapy (Vissers et al. 2007).

Numerous extensions at the apical pole membrane suggest greatly increased tendency for endothelial microparticle formation (Lynch and Ludlam 2007). In fact, their increased production of EC-derived microparticles has been demonstrated (JM Aerts, unpublished observations), especially in splenectomized GD patients. In patients with splenomegaly, increased clearance of microparticles by the enlarged spleen most likely explains the relatively low levels of circulating EC-derived microparticles. 
In the series of Fabry hemizygotes, the blood capillaries manifested typical storage processes in ECs as well as in pericytes. The ECs engorged with lysosomal storage material in this setting displayed cytoplasmic enlargement proportional to the degree of lysosomal storage. In Fabry disease, alterations of apical and basal cell membranes are not found in contrast to those present in our GD patients. The apical membrane projections were present but only exceptionally prominent. The subendothelial basement membranes were frequently multiplied. In angiokeratoma of Fabry disease, in contrast, ECs were thin, despite the presence of storage lysosomes (Elleder 2003). It would be instructive to compare endothelial function and morphology in GD with Fabry disease. In the mouse model of Fabry disease, signs of EC dysfunction have been described (Eitzman et al. 2003; Park et al. 2008). Moreover, in Fabry disease patients, enzyme replacement therapy led to a decrease in circulating endothelial microparticles (Gelderman et al. 2007). A recent survey of a cohort of 36 Fabry disease patients, however, revealed only minimal abnormalities in endothelial microparticles, and of markers for platelet and endothelial activation, coagulation activation and fibrinolysis. The earlier reported abnormalities in severely affected Fabry disease patients seem to be better explained by renal insufficiency than Fabry disease itself (Vedder et al. 2009 ).

In GD patients, EC changes dominated over those seen in pericytes, which were changed to a lesser degree. However, there were definite signs of their activation, and it is important to keep in mind that their activation may be connected with calcifications (Armulik et al. 2005), repeatedly seen, e.g., in GD II (Mignot et al. 2003; Sherer et al. 1993; Spear et al. 2007).

Our results are descriptive but make a compelling case for further study of ECs in GD. The unique morphological pattern in individual capillaries taken together with remarkable tendency to EC loose anchoring as well as their protrusion into the lumen argues strongly for a pathophysiologic role of resident ECs and their possible increased exchange within the pool of circulating EC precursors (Hristov et al. 2003; Xu 2007).

\section{Conclusion and perspectives}

We are well aware of the limitations of our study, which is restricted to the thus far nontraditional type of bioptic sample. However, interest should concentrate on skin biopsies in further cases and types of GD, first to nonneuronopathic GD type I (before and during enzyme replacement theraphy), and to the capillary network generally, as this may open and define a novel field in molecular cell pathophysiology of the disorder: endothelial dysfunction.
Acknowledgment Dr. Věra Malinová, Charles University, 1st Faculty of Medicine and General Teaching Hospital, Department of Pediatrics and Adolescent Medicine, Prague, Czech Republic, provided clinical data of patient 1. Dr. F. Stehling, Department of Neuropediatrics, Universitätsklinikum, Essen, Germany, is thanked for providing the skin biopsy and clinical data of patient 2; and Dr. E. Mengel, Center for Lysosomal Diseases, University of Mainz, Germany, for providing neuroophthalmologic data of that patient.

The study was supported by a research project of the Ministry of Education Youth and Sports Czech Republic (Grant No. MSM 0021620806).

Open Access This article is distributed under the terms of the Creative Commons Attribution Noncommercial License which permits any noncommercial use, distribution, and reproduction in any medium, provided the original author(s) and source are credited.

\section{References}

Adar T, Ben-Ami R, Elstein D et al (2008) Increased red blood cell aggregation in patients with Gaucher disease is noninflammatory. Clin Hemorheol Microcirc 40:113-118

Aerts JM, Hollak CE (1997) Plasma and metabolic abnormalities in Gaucher's disease. Baillieres Clin Haematol 10:691-709

Agha-Majzoub R, Becker RP, Schraufnagel DE et al (2005) Angiogenesis: the major abnormality of the keratin-14 IL-4 transgenic mouse model of atopic dermatitis. Microcirculation 12:455-476

Armulik A, Abramsson A, Betsholtz C (2005) Endothelial/pericyte interactions. Circ Res 97:512-523

Beutler E, Grabowski GA (2001) Gaucher disease. In: Scriver CR, Beaudet AL, Sly WS, Valle D (eds) The metabolic and molecular bases of inherited disease. McGraw-Hill, New York, pp 3635-3668

Boot RG, Verhoek M, Donker-Koopman W et al (2007) Identification of the non-lysosomal glucosylceramidase as beta-glucosidase 2. J Biol Chem 282:1305-1312

Boven LA, van Meurs M, Boot RG et al (2004) Gaucher cells demonstrate a distinct macrophage phenotype and resemble alternatively activated macrophages. Am J Clin Pathol 122:359-369

Burri PH, Djonov V (2002) Intussusceptive angiogenesis-the alternative to capillary sprouting. Mol Aspects Med 23:S1-S27

Carpenter S (1988) Morphological diagnosis and misdiagnosis in Batten-Kufs disease. Am J Med Genet Suppl 5:85-91

Chen M, Wang J (2008) Gaucher disease: review of the literature. Arch Pathol Lab Med 132:851-853

Cogolludo A, Moreno L, Frazziano G et al (2009) Activation of neutral sphingomyelinase is involved in acute hypoxic pulmonary vasoconstriction. Cardiovasc Res 82:296-302

Cox TM (2005) Biomarkers in lysosomal storage diseases: a review. Acta Paediatr Suppl 94:39-42, discussion 37-38

Deegan PB, Moran MT, McFarlane I et al (2005) Clinical evaluation of chemokine and enzymatic biomarkers of Gaucher disease. Blood Cells Mol Dis 35:259-267

Desnick RJ, Gatt S, Grabowski GA (1982) Gaucher disease: a century of delineation and research. Alan R. Liss, New York

Djonov V, Baum O, Burri PH (2003) Vascular remodeling by intussusceptive angiogenesis. Cell Tissue Res 314:107-117

Dye J, Lawrence L, Linge C et al (2004) Distinct patterns of microvascular endothelial cell morphology are determined by extracellular matrix composition. Endothelium 11:151-167

Eblan MJ, Goker-Alpan O, Sidransky E (2005) Perinatal lethal Gaucher disease: a distinct phenotype along the neuronopathic continuum. Fetal Pediatr Pathol 24:205-222 
Eitzman DT, Bodary PF, Shen Y et al (2003) Fabry disease in mice is associated with age-dependent susceptibility to vascular thrombosis. J Am Soc Nephrol 14:298-302

Elleder M (2003) Sequelae of storage in Fabry disease-pathology and comparison with other lysosomal storage diseases. Acta Paediatr Suppl 92:46-53, discussion 45

Elleder M (2006) Glucosylceramide transfer from lysosomes-the missing link in molecular pathology of glucosylceramidase deficiency: a hypothesis based on existing data. J Inherit Metab Dis 29:707-715

Elstein D, Renbaum P, Levy-Lahad E et al (2000) Incidence of thrombophilia in patients with Gaucher disease. Am J Med Genet 95:429-431

Elstein D, Nir A, Klutstein M et al (2005) C-reactive protein and NTproBNP as surrogate markers for pulmonary hypertension in Gaucher disease. Blood Cells Mol Dis 34:201-205

Fujimoto A, Onodera H, Mori A et al (2004) Vascular endothelial growth factor reduces mural cell coverage of endothelial cells and induces sprouting rather than luminal division in an HT1080 tumour angiogenesis model. Int J Exp Pathol 85:355-364

Gelderman MP, Schiffmann R, Simak J (2007) Elevated endothelial microparticles in Fabry children decreased after enzyme replacement therapy. Arterioscler Thromb Vasc Biol 27: e138-e139

Ghauharali-van der Vlugt K, Langeveld M, Poppema A et al (2008) Prominent increase in plasma ganglioside GM3 is associated with clinical manifestations of type I Gaucher disease. Clin Chim Acta 389:109-113

Goldblatt J, Beighton P (1984) Cutaneous manifestations of Gaucher disease. Br J Dermatol 111:331-334

Groener JE, Poorthuis BJ, Kuiper S et al (2008) Plasma glucosylceramide and ceramide in type 1 Gaucher disease patients: correlations with disease severity and response to therapeutic intervention. Biochim Biophys Acta 1781:72-78

Hartree EF (1972) Determination of protein: a modification of the Lowry method that gives a linear photometric response. Anal Biochem 48:422-427

Hein LK, Duplock S, Hopwood JJ et al (2008) Lipid composition of microdomains is altered in a cell model of Gaucher disease. $\mathrm{J}$ Lipid Res 49:1725-1734

Hodanova K, Hrebicek M, Cervenkova M et al (1999) Analysis of the beta-glucocerebrosidase gene in Czech and Slovak Gaucher patients: mutation profile and description of six novel mutant alleles. Blood Cells Mol Dis 25:287-298

Hollak CE, Evers L, Aerts JM et al (1997a) Elevated levels of M-CSF, sCD14 and IL8 in type 1 Gaucher disease. Blood Cells Mol Dis 23:201-212

Hollak CE, Levi M, Berends F et al (1997b) Coagulation abnormalities in type 1 Gaucher disease are due to low-grade activation and can be partly restored by enzyme supplementation therapy. Br J Haematol 96:470-476

Holleran WM, Ginns EI, Menon GK et al (1994a) Consequences of beta-glucocerebrosidase deficiency in epidermis. Ultrastructure and permeability barrier alterations in Gaucher disease. J Clin Invest 93:1756-1764

Holleran WM, Takagi Y, Menon GK et al (1994b) Permeability barrier requirements regulate epidermal beta-glucocerebrosidase. J Lipid Res 35:905-912

Holleran WM, Ziegler SG, Goker-Alpan O et al (2006) Skin abnormalities as an early predictor of neurologic outcome in Gaucher disease. Clin Genet 69:355-357

Hristov M, Erl W, Weber PC (2003) Endothelial progenitor cells: mobilization, differentiation, and homing. Arterioscler Thromb Vasc Biol 23:1185-1189

Lee RE (1982) The pathology of Gaucher disease. In: Desnick RJ, Gatt S, Grabowski GA (eds) Gaucher disease: a century of delineation and research. Alan R. Liss, New York, pp $177-$ 218

Lee RE (2007) Pathologic anatomy of Gaucher disease: a pictorial essay. In: Futerman AH, Zimran A (eds) Gaucher disease. CRC Press, Boca Raton, pp 197-223

Liptak P, Kemeny E, Morvay Z et al (2005) Peritubular capillary damage in acute humoral rejection: an ultrastructural study on human renal allografts. Am J Transplant 5:2870-2876

Lynch SF, Ludlam CA (2007) Plasma microparticles and vascular disorders. Br J Haematol 137:36-48

Martin S, Pitcher D, Tschen J et al (1980) Reactive angioendotheliomatosis. J Am Acad Dermatol 2:117-123

Mignot C, Gelot A, Bessieres B et al (2003) Perinatal-lethal Gaucher disease. Am J Med Genet A 120A:338-344

Michelakakis H, Spanou C, Kondyli A et al (1996) Plasma tumor necrosis factor-a (TNF-a) levels in Gaucher disease. Biochim Biophys Acta 1317:219-222

Norrby K (1997) Angiogenesis: new aspects relating to its initiation and control. Apmis 105:417-437

Park JL, Whitesall SE, D'Alecy LG et al (2008) Vascular dysfunction in the alpha-galactosidase A-knockout mouse is an endothelial cell-, plasma membrane-based defect. Clin Exp Pharmacol Physiol 35:1156-1163

Pavelka M, Roth J (2005) Functional ultrastructure. An atlas of tissue biology and pathology. Springer Verlag, Wien

Planes C, Valeyre D, Loiseau A et al (1994) Ultrastructural alterations of the air-blood barrier in sarcoidosis and hypersensitivity pneumonitis and their relation to lung histopathology. Am J Respir Crit Care Med 150:1067-1074

Polosukhin VV (1997) Dynamics of the ultrastructural changes in blood and lymphatic capillaries of bronchi in inflammation and following endobronchial laser therapy. Virchows Arch 431:283-290

Polverini PJ (1995) The pathophysiology of angiogenesis. Crit Rev Oral Biol Med 6:230-247

Reissner K, Tayebi N, Stubblefield BK et al (1998) Type 2 Gaucher disease with hydrops fetalis in an Ashkenazi Jewish family resulting from a novel recombinant allele and a rare splice junction mutation in the glucocerebrosidase locus. Mol Genet Metab 63:281-288

Risau W (1997) Mechanisms of angiogenesis. Nature 386:671-674

Sharma R, Hudak ML, Perszyk AA et al (2000) Perinatal lethal form of Gaucher's disease presenting with hemosiderosis. Am J Perinatol 17:201-206

Sherer DM, Metlay LA, Sinkin RA et al (1993) Congenital ichthyosis with restrictive dermopathy and Gaucher disease: a new syndrome with associated prenatal diagnostic and pathology findings. Obstet Gynecol 81:842-844

Shyong EQ, Gorevic P, Lebwohl M et al (2002) Reactive angioendotheliomatosis and sarcoidosis. Int J Dermatol 41:894-897

Sidransky E, Sherer DM, Ginns EI (1992) Gaucher disease in the neonate: a distinct Gaucher phenotype is analogous to a mouse model created by targeted disruption of the glucocerebrosidase gene. Pediatr Res 32:494-498

Spear GS, Beutler E, Hungs M (2007) Congenital Gaucher disease with nonimmune hydrops/erythroblastosis, infantile arterial calcification, and neonatal hepatitis/fibrosis. Clinicopathologic report with enzymatic and genetic analysis. Fetal Pediatr Pathol 26:153-168

Stone DL, van Diggelen OP, de Klerk JB et al (1999) Is the perinatal lethal form of Gaucher disease more common than classic type 2 Gaucher disease? Eur J Hum Genet 7:505-509

Takemura T, Shishiba T, Akiyama O et al (1997) Vascular involvement in cutaneous sarcoidosis. Pathol Int 47:84-89

Tsukada N, Yanagisawa N, Mochizuki I (1995) Endothelial cell damage in sarcoidosis and neurosarcoidosis: autoantibodies to endothelial cells. Eur Neurol 35:108-112 
Van Gysel D, Lijnen RL, Moekti SS et al (2002) Collodion baby: a followup study of 17 cases. J Eur Acad Dermatol Venereol 16:472-475

van Weely S, Brandsma M, Strijland A et al (1993) Demonstration of the existence of a second, non-lysosomal glucocerebrosidase that is not deficient in Gaucher disease. Biochim Biophys Acta 1181:55-62

Vedder AC et al. (2009) Plasma markers of coagulation and endothelial activation in Fabry disease: impact of renal impairment. Nephrol Dial Transplant 24(10):3074-3081

Vissers JP, Langridge JI, Aerts JM (2007) Analysis and quantification of diagnostic serum markers and protein signatures for Gaucher disease. Mol Cell Proteomics 6:755-766

Vracko R, Benditt EP (1974) Manifestations of diabetes mellitus-their possible relationships to an underlying cell defect. A review. Am J Pathol 75:204-224
Walski M, Frontczak-Baniewicz M (2003) New vessel formation after surgical brain injury in the rat's cerebral cortex II. Formation of the blood vessels distal to the surgical injury. Acta Neurobiol Exp (Wars) 63:77-82

Wenger D, Williams C (1991) Screening for lysosomal disorders. In: Hommes $\mathrm{F}$ (eds). Techniques in diagnostic human biochemical genetics. Willey-Liss, New York, pp 587-617

Xu Q (2007) Progenitor cells in vascular repair. Curr Opin Lipidol 18:534-539

Yildiz Y, Matern H, Thompson B et al (2006) Mutation of betaglucosidase 2 causes glycolipid storage disease and impaired male fertility. J Clin Invest 116:2985-2994

Zhou AL, Egginton S, Brown MD et al (1998) Capillary growth in overloaded, hypertrophic adult rat skeletal muscle: an ultrastructural study. Anat Rec 252:49-63 